\title{
Review of: "Remodeling tumor microenvironment by liposomal codelivery of DMXAA and simvastatin inhibits malignant melanoma progression"
}

Sankar Bhattacharyya

Potential competing interests: The author(s) declared that no potential competing interests exist.

In this article authors has proposed that liposomal codelivery of MXAA and simvastatin was capable of decreasing melanoma tumor mass and influence tumor microenvironment. Antibody Array experiments with tumor lysate clearly shows level of different pro-angiogenic and inflammatory gene expression can be suppressed by combination drug treatment ( DMAXAA+ SIM). Although in the title authors indicated that this treatment remodels tumor microenvironment and re-educates tumor associated macrophage more towards M1 phenotype but direct remodelling and TAM re-education activities are not clear from experiments. Observed effect could be due to the fact that treatment reduces tumor volume directly leading to less stress on system and fewer tumor cells thus abrogating pro-inflammatory microenvironment resulting in slight shift towards M1 phenotype of tumor site macrophages. In Figure number $2 A$ and $B$, inos and argenase level in untreated tumor associated macrophage should have been incorporated rather than using control group just as calibrator, in that case control. tumor free skin resident macrophages could have been used as calibrator.

Declaring shift in macrophage phenotype just based on iNOS and argenase may not be sufficient as TAMs are extremely plastic with a number of markers that should have been tested, for example IL10 and IL12 ratio in treated and untreated TAMS, MHCII, CD86, CD206 etc. Also microenvironment was not directly assessed in this article, changing level of proteins in antibody array in tumor lysate may not directly transpire to stromal cells and cancer associated fibroblasts. In figure 4 authors explored pro and antiapoptotic protein status in treated and control tumor. Again this portion could have been explored in detail, mechanism of apoptosis as well as direct apoptotic indicators such as TUNEL assay or annexin $v$ staining should have been undertaken. Again in figure 6, effect of treatment of HIF1 was approached rather halfheartedly, it is not clear whether actual nuclear localization of HIF1 or HIFlalpha was reduced or not. Over all this reviewer feel that authors tried to address too many questions without focusing on thoroughly resolving any, Experiments could have been designed more specifically to resolve mechanism. As a whole, even with impressive number of experiments and arrays used by the authors this article still provides preliminary results and detail study of the mechanisms of observed effects should be undertaken. 
